\title{
Estudo do comportamento cinético de oxidação do ácido mandélico por vanádio(V) em meio de ácido sulfúrico: efeito da presença de CTAB.
}

\author{
Study of the kinetic behavior of mandelic acid oxidation by \\ $\operatorname{vanadium}(\mathbf{V})$ in sulfuric acid medium: effect of $C T A B$ presence.
}

\author{
Eduardo Niehues ${ }^{1}$; Rômulo Augusto Ando'; Keiko Takashima ${ }^{2}$
}

Resumo

O comportamento cinético da oxidação de ácido mandélico por vanádio(V) em meio de ácido sulfúrico foi investigado a $303 \mathrm{~K}$. A constante de velocidade foi determinada espectrofotometricamente, por meio do aparecimento de vanádio(IV) em $760 \mathrm{~nm}$. A oxidação mostrou dependência de primeira ordem com relação à concentração de vanádio(V) e ordem fracionária com relação à concentração de ácido mandélico e de ácido sulfúrico. A lei de velocidade não foi afetada na presença do surfactante catiônico brometo de cetil-trimetil-amônio. A constante de velocidade foi sistematicamente maior em meio contendo surfactante. A constante de velocidade aumentou com o aumento da força iônica e com a diminuição da constante dielétrica. Os parâmetros de ativação foram calculados a partir das constantes de velocidade de $2^{\mathrm{a}}$ ordem, $k_{2}$, para diferentes concentrações de substrato de 298 a $318 \mathrm{~K}$. O mecanismo de oxidação foi proposto a partir da lei de velocidade experimental.

Palavras-chave: Ácido mandélico. $\alpha$-hidroxiácido.Oxidação por vanádio(V)M.

\begin{abstract}
The mandelic acid oxidation by vanadium (V) in sulfuric acid medium was studied at $303 \mathrm{~K}$. The reaction rate was determined spectrophotometrically by means of the formation of vanadium (IV) at $760 \mathrm{~nm}$. The oxidation reaction showed first order dependence in terms of vanadium (V)

${ }^{1}$ Bolsista de Iniciação Científica PIBIC/CNPq.

${ }^{2}$ Departamento de Química/CCE/UEL. E-mail: keiko@uel.br.
\end{abstract}

Semina: Ciências Exatas e Tecnológicas, Londrina, v. 27, n. 2, p. 183-191, jul./dez. 2006 
concentration and fraction order concerning mandelic acid and sulfuric acid concentrations. The rate law was not influenced by the presence of cetil trimethyl ammonium bromide cationic surfactant. The rate constant increased with the increase of the ionic strength and with the decrease of the dielectric constant. The activation parameters were calculated based on the rate constants determined in the 298 to $318 \mathrm{~K}$ interval. The oxidation mechanism was proposed based on the experimental rate law.

Key words: Mandelic acid. á-hydroxyacid. Oxidation by vanadium (V).

\section{Introdução}

A redução de vanádio(V) tem sido investigada sob a cinética de primeira ordem, por meio da formação de vanádio(IV). Tem sido mostrado que a oxidação de compostos orgânicos (BRUYÈRE et al, 2001; KHAN; BABU; DIN, 2004) ocorre via mecanismo radicalar, no qual o vanádio(V) sofre a redução monoeletrônica (LITTLER, WATERS, 1959). Devido às analogias entre as catálises micelar e enzimática, os substratos orgânicos têm sido oxidados na presença de surfactantes, para investigar a sua influência na velocidade de reação (DIN; MORSHED; KHAN, 2002; PANDEY; UPADHYAY, 2005). Estudos anteriores realizados por este grupo (ANDO et al., 2003; RAMINELLI; BARRETO; TAKASHIMA, 2000; TAKASHIMA; ZIGLIO; RONCONI, 1998; ZIGLIO; TAKASHIMA, $1995 ; \quad$ CLEMENTIN; TAKASHIMA, 1993; TAKASHIMA, 1993) acerca da oxidação de $\alpha$-hidroxiácidos por vanádio(V) em solução aquosa, têm mostrado que a estrutura e a natureza, tanto do substrato quanto do oxidante, a capacidade de oxidação do substrato, a força iônica da mistura de reação, além de outras condições experimentais, podem influenciar a cinética e o mecanismo de oxidação desses compostos. $\mathrm{O}$ vanádio(V) em solução ácida ocorre como íon pervanadila $\left(\mathrm{VO}_{2}^{+}\right)$e pode ser mais ou menos reativo dependendo da natureza do meio. Espécies como $\mathrm{VO}(\mathrm{OH})^{2+}$ e $\mathrm{V}(\mathrm{OH})_{3}{ }^{2+}$ ocorrem em solução aquosa de ácido perclórico, enquanto $\mathrm{V}(\mathrm{OH})_{3} \mathrm{HSO}_{4}{ }^{+} \mathrm{e}$ $\mathrm{V}(\mathrm{OH})_{2}\left(\mathrm{HSO}_{4}\right)_{2}{ }^{+}$ocorrem em meio de ácido sulfúrico (MEHROTRA, 1968). A participação destas espécies nas oxidações tem mostrado que diferentes mecanismos podem ser atribuídos, dependendo do meio em que seja realizada a oxidação.

Os ácidos $\alpha$-hidroxicarboxílicos ou a-hidroxiácidos são compostos bifuncionais que apresentam em suas estruturas, pelo menos, um grupo carbonila e dois hidroxílicos, bastante solúveis em água e vêm sendo utilizados nas últimas décadas como ingrediente na composição de cosméticos para tratamento de rejuvenescimento da pele humana. São geralmente encontrados e isolados de frutas de muitas espécies. O ácido málico é encontrado em maçãs; o ácido cítrico pode ser isolado de frutas cítricas; o ácido glicólico da cana-de-açúcar e mel de abelha; o ácido lático é encontrado em leite coalhado e o ácido tartárico pode ser isolado de uva fermentada. Além desses compostos, o ácido $\alpha$-hidroxifenil acético ou ácido mandélico (Figura 1) tem sido usado para a mesma finalidade. Apesar de menos solúvel em água devido ao núcleo benzênico em sua estrutura, este substrato tem tido a preferência sobre ácido lático ou ácido glicólico por não ser irritante à pele.

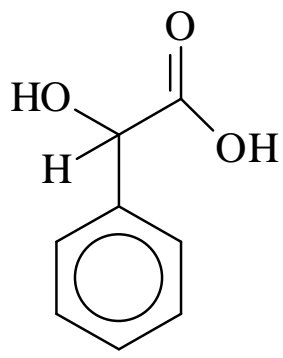

Figura 1 - Estrutura química do ácido mandélico. 
Dando continuidade aos estudos sobre o comportamento cinético de $\alpha$-hidroxiácidos por vanádio(V) em meio ácido, relatamos os efeitos do surfactante catiônico brometo de cetil trimetil amônio (CTAB) sobre a oxidação do ácido mandélico por vanádio(V) em meio de ácido sulfúrico a $303 \mathrm{~K}$.

\section{Procedimento Experimental}

Todos os reagentes utilizados neste trabalho foram de grau analítico e as soluções foram preparadas com água purificada (Elga USF). As soluções de vanádio $(\mathrm{V}), \mathrm{V}(\mathrm{V})$, foram preparadas, como descritas previamente (ZIGLIO; TAKASHIMA, 1995; CLEMENTIN; TAKASHIMA, 1993), adicionandose concentrações adequadas de ácido sulfúrico em vanadato de amônio, $\mathrm{NH}_{4} \mathrm{VO}_{3}$ (Merck). A força iônica da solução foi controlada adicionando-se à solução de vanádio(V), concentração adequada de bissulfato de sódio, $\mathrm{NaHSO}_{4}$ (Nuclear). O surfactante catiônico brometo de cetil trimetil amônio, CTAB (Vetec) foi usado como recebido.

Todas as medidas cinéticas foram realizadas sob condições de pseudo- $1^{\text {a }}$ ordem com excesso de, pelo menos, 50 vezes de ácido mandélico sobre o vanádio(V). A formação do vanádio(IV) foi acompanhada por 4 a 5 tempos de meia vida, medindo-se a absorvância em $760 \mathrm{~nm}(\varepsilon=18.91 \pm 0,83$ mol L ${ }^{-1} \mathrm{~cm}^{-1}$ ) e $303 \mathrm{~K}$ mantendo-se constante a temperatura no intervalo de $\pm 0,1^{\circ} \mathrm{C}$, através da circulação de água proveniente do banho termostático (Microquímica, MQBTC 99-20). Os experimentos foram realizados em duplicatas e quando necessário em triplicatas.

A constante de velocidade de reação de pseudo$1^{\mathrm{a}}$. ordem $\left(k_{o b s}\right)$ foi calculada através da inclinação do gráfico entre o ln da concentração de V(IV) em função do tempo, utilizando o programa Datalink instalado no microcomputador acoplado ao espectrofotômetro UV-Vis (Hitachi U3000). A mediação de radicais livres na oxidação; a produção de $\mathrm{CO}_{2}$; a caracterização de benzaldeído como um dos produtos de oxidação, foram previamente descritos (CLEMENTIN; TAKASHIMA, 1993; TAKASHIMA, 1993). A reação total para a oxidação de ácido mandélico por vanádio(V) em meio de ácido sulfúrico pode ser representada pela seguinte equação:

$2 \mathrm{~V}(\mathrm{~V})+\mathrm{C}_{6} \mathrm{H}_{5} \mathrm{CH}(\mathrm{OH}) \mathrm{COOH} \rightarrow 2 \mathrm{~V}(\mathrm{IV})+$ $\mathrm{C}_{6} \mathrm{H}_{5} \mathrm{COH}+\mathrm{CO}_{2}+2 \mathrm{H}_{2} \mathrm{O}$

Nesta equação, $V(V)$ e $V(I V)$ representam respectivamente as espécies de vanádio positivamente carregadas, $\mathrm{V}(\mathrm{OH})_{3} \mathrm{HSO}_{4}^{+} \mathrm{e} \mathrm{V}(\mathrm{OH})_{2} \mathrm{HSO}_{4}^{+}$.

\section{Resultados \\ Efeito da concentração dos reagentes}

A concentração de ácido mandélico, [HMd], foi variada de 0,25 a $1,25 \mathrm{~mol} \mathrm{~L}^{-1}$ a $303 \mathrm{~K}$ mantendo-se constantes as concentrações iniciais de vanádio(V), $[\mathrm{V}(\mathrm{V})]_{\mathrm{o}}, \mathrm{em} 1,0 \times 10^{-2} \mathrm{~mol} \mathrm{~L}^{-1},\left[\mathrm{H}_{2} \mathrm{SO}_{4}\right]_{\mathrm{o}}, 0,1 \mathrm{~mol} \mathrm{~L}^{-1}$, na ausência e na presença de $[\mathrm{CTAB}]_{\mathrm{o}} 2,5 \times 10^{-2} \mathrm{~mol} \mathrm{~L}^{-1}$. A constante de velocidade, $k_{o b s}$, foi maior em meio de surfactante e aumentou linearmente com o aumento da concentração de HMd como pode ser observada na Figura 2. A constante de velocidade praticamente triplicou $\left(1,49 \times 10^{-2}\right.$ a $\left.4,32 \times 10^{-2} \mathrm{~s}^{-1}\right)$ na ausência de surfactante, e resultou em uma ordem de reação de 0,68 ( $r=0,999)$ (Figura 2a). Na presença de CTAB, o aumento foi menos acentuado em concentrações maiores no intervalo mencionado $\left(2,07 \times 10^{-2}\right.$ a $4,77 \times 10^{-2}$ $\left.\mathrm{s}^{-1}\right)$, dando uma ordem de $0,51(r=0,998)$. A dependência de $1^{\mathrm{a}}$. ordem com relação a $[\mathrm{V}(\mathrm{V})] \mathrm{em}$ meio de ácido sulfúrico foi inferida, porque os gráficos individuais de ln da concentração de vanádio(V), [V(V)], em função do tempo para diferentes $[\mathrm{HMd}]_{0}$ mostraram linearidade satisfatória $(r=0,997)$ a $303 \mathrm{~K}$. A variação de $0,50 \times 10^{-2}$ a $2,50 \times 10^{-2} \mathrm{~mol} \mathrm{~L}^{-1}$ para vanádio(V), fixando-se as concentrações iniciais de HMd em 0,5 mol L-1 e de $\mathrm{H}_{2} \mathrm{SO}_{4}$ em $0,1 \mathrm{~mol} \mathrm{~L}^{-1}$ diminuiu a constante de velocidade de $2,45 \times 10^{-2}$ a $2,28 \times 10^{-2} \mathrm{~s}^{-1}$ na ausência de surfactante (Figura 2b). Na presença de CTAB $2,5 \times 10^{-2} \mathrm{~mol} \mathrm{~L}^{-1}$, a constante de velocidade foi relativamente maior, tendo-se o valor de $3,24 \times 10^{-2} \mathrm{~s}^{-1}$ em $0,50 \times 10^{-2} \mathrm{molL}^{-1} \mathrm{de} \mathrm{V}(\mathrm{V})$ a $3,04 \times 10^{-2} \mathrm{~s}^{-1} \mathrm{em} 1,50 \times 10^{-}$ ${ }^{2} \mathrm{~mol} \mathrm{~L}^{-1} \mathrm{e}$ aumentando até $3,38 \times 10^{-2} \mathrm{~s}^{-1} \mathrm{em} 2,5 \times 10^{-2}$ mol L L de vanádio(V) a $303 \mathrm{~K}$. 


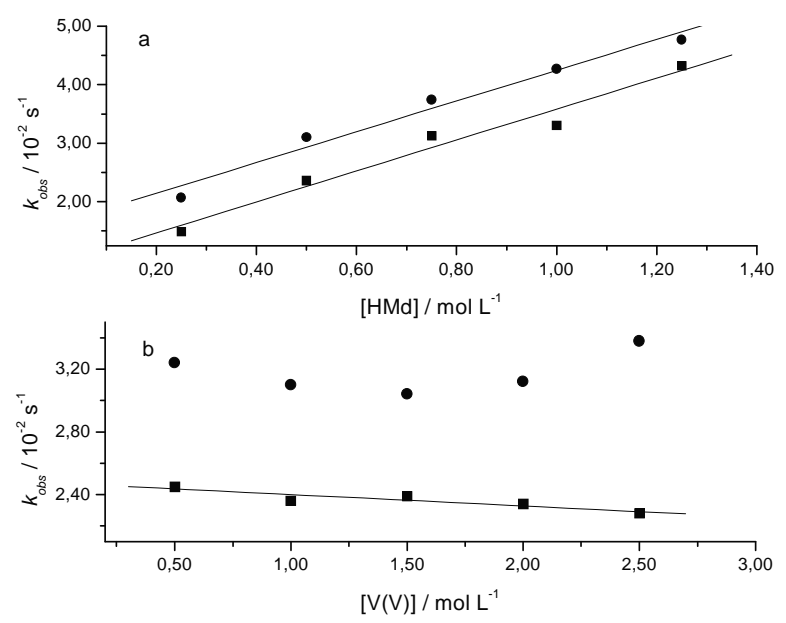

Figura 2 - Efeitos das concentrações de ácido mandélico (a) e de vanádio(V) (b) sobre a constante de velocidade $k_{\text {obs }}$ na ausência ( $\left.\mathbf{\square}\right)$ e presença $(\bullet)$ de CTAB a $303 \mathrm{~K}$ para $\left[\mathrm{H}_{2} \mathrm{SO}_{4}\right]_{0} 0,10 \mathrm{~mol} \mathrm{~L}^{-1}$.

\section{Efeito da Concentração do Íon Hidrogênio}

A adição de ácido sulfúrico de 0,05 a $0,30 \mathrm{~mol} \mathrm{~L}^{-}$ ${ }^{1}$ a $303 \mathrm{~K}$, mantendo-se fixas as concentrações iniciais, $[\mathrm{HMd}]_{\mathrm{o}} 0,5 \mathrm{~mol} \mathrm{~L}^{-1} \mathrm{e}[\mathrm{V}(\mathrm{V})]_{\mathrm{o}} 1,0 \times 10^{-2} \mathrm{~mol}$ $\mathrm{L}^{-1}$, aumentou a constante de velocidade de oxidação conforme pode ser observada na Figura 3. O aumento da constante de velocidade de $1,65 \times 10^{-2}$ a $4,24 \times 10^{-2}$ $\mathrm{s}^{-1}$ na ausência de CTAB, resultou em uma ordem de $0,52(r=0,999)$. As constantes de velocidade foram maiores na presença de CTAB $2,5 \times 10^{-2} \mathrm{~mol} \mathrm{~L}^{-1}$, mas o aumento relativo foi menor, já que a variação ocorreu de $2,41 \times 10^{-2}$ a $5,51 \times 10^{-2} \mathrm{~s}^{-1}$ dando uma ordem de $0,46(r=0,995)$.

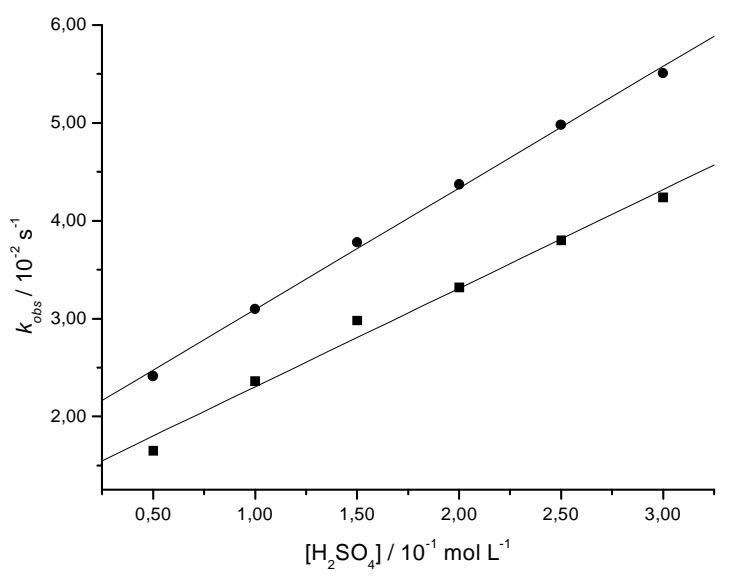

Figura 3 - Efeito da concentração de $\mathrm{H}_{2} \mathrm{SO}_{4}$ sobre a constante de velocidade a $303 \mathrm{~K}$ na ausência ( $\bullet$ ) e presença ( • ) de CTAB para $[\mathrm{V}(\mathrm{V})]_{\mathrm{o}} 1,0 \times 10^{-2} \mathrm{~mol} \mathrm{~L}^{-1} \mathrm{e}[\mathrm{HMd}]_{\mathrm{o}} 0,50 \mathrm{~mol} \mathrm{~L}^{-1}$ a $303 \mathrm{~K}$.

\section{Efeito da Força Iônica}

O efeito da força iônica foi estudado adicionandose bissulfato de sódio como eletrólito, de 0,11 a 1,00 mol L $\mathrm{L}^{-1}$ a $303 \mathrm{~K}$, mantendo-se constantes as demais concentrações iniciais (Figura 4). Os gráficos de log de $k_{\text {obs }}$, em função da raiz quadrada da força iônica em $303 \mathrm{~K}$, resultaram em produtos iônicos, $\mathrm{Z}_{+} \mathrm{Z}$, respectivamente iguais a $0,54(r=0,998)$ e $0,43(r=$ $0,996)$ na presença e na ausência de CTAB. A presença de CTAB diminuiu o efeito da força iônica na constante de velocidade, quando comparada com aquela sem o surfactante, conforme pode ser observada na Figura 4.

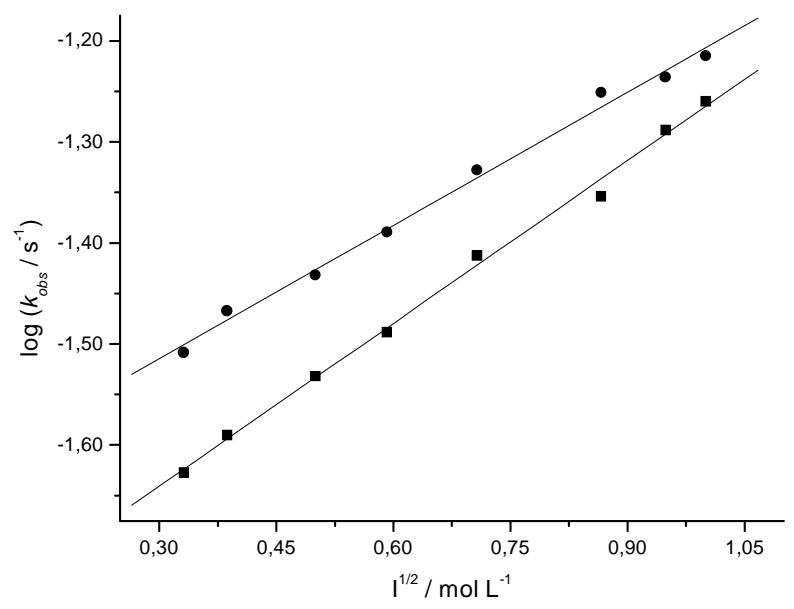

Figura 4 - Efeito da força iônica sobre a constante de velocidade de pseudo- $1^{\text {a }}$ ordem a $303 \mathrm{~K}$ na ausência $(\boldsymbol{\square})$ e presença $(\bullet)$ de surfactante CTAB para $[\mathrm{HMd}]_{0} 0,50 \mathrm{~mol} \mathrm{~L}^{-1},[\mathrm{~V}(\mathrm{~V})]_{\mathrm{o}} 1,00 \times 10^{-2}$ $\mathrm{mol} \mathrm{L}^{-1} \mathrm{e}\left[\mathrm{H}_{2} \mathrm{SO}_{4}\right]_{\mathrm{o}} 0,10 \mathrm{~mol} \mathrm{~L}^{-1}$

\section{Efeito do CTAB}

O efeito da adição de CTAB de 1,0x10-2 a 10,0x10${ }^{2} \mathrm{~mol} \mathrm{~L}^{-1}$ a $303 \mathrm{~K}$ foi investigado fixando-se ou não a força iônica na oxidação de ácido mandélico para $[\mathrm{HMd}]_{\mathrm{o}} 0,5 \mathrm{~mol} \mathrm{~L}^{-1}$ e $[\mathrm{V}(\mathrm{V})]_{\mathrm{o}} 1,0 \times 10^{-2} \mathrm{~mol} \mathrm{~L}^{-1}$, conforme mostrado na Tabela 1. A partir desta tabela, observou-se que a oxidação é mais rápida com controle de força iônica de $0,35 \mathrm{~mol} \mathrm{~L}^{-1}$ e na presença de CTAB 2,5x10-2 $\mathrm{mol} \mathrm{L}^{-1}$. 
Tabela 1 - Efeito da concentração de CTAB sobre a constante de velocidade de pseudo- $1^{\mathrm{a}}$ ordem a $303 \mathrm{~K}$ para força iônica de 0,11 mol $\mathrm{L}^{-1}$ e de $0,35 \mathrm{~mol} \mathrm{~L}^{-1}:[\mathrm{HMd}]_{\mathrm{o}}=0,50 \mathrm{~mol} \mathrm{~L}^{-1},[\mathrm{~V}(\mathrm{~V})]_{\mathrm{o}}=1,00 \times 10^{-2} \mathrm{~mol} \mathrm{~L}^{-1} \mathrm{e}\left[\mathrm{H}_{2} \mathrm{SO}_{4}\right]_{\mathrm{o}}=0,10 \mathrm{~mol} \mathrm{~L}^{-1}$.

\begin{tabular}{ccc}
\hline$[\mathrm{CTAB}] / 10^{-2} \mathrm{~mol} \mathrm{~L}^{-1}$ & $I / \mathrm{mol} \mathrm{L}^{-1}$ & $k_{o b s} / 10^{-2} \mathrm{~s}^{-1}$ \\
\hline 1,0 & 0,11 & 2,90 \\
2,5 & 0,11 & 3,10 \\
5,0 & 0,11 & 3,18 \\
7,5 & 0,11 & 3,37 \\
10,0 & 0,11 & 3,41 \\
1,0 & 0,35 & 3,29 \\
2,5 & 0,35 & 3,64 \\
5,0 & 0,35 & 3,71 \\
7,5 & 0,35 & 3,90 \\
10,0 & 0,35 & 4,09 \\
\hline
\end{tabular}

\section{Efeito do Solvente}

Oefeito do solvente foi investigado por meio da mistura contendo diferentes proporções de metanol (0 a 40\%) em água a $303 \mathrm{~K}$ com ou sem controle da força iônica na presença e na ausência de CTAB $2,5 \times 10^{-2} \mathrm{~mol} \mathrm{~L}^{-1}$. Observou-seque, quando a força iônica não foi controlada, ou seja, com forçaiônica inicial de $0,11 \mathrm{~mol} \mathrm{~L}^{-1}$ na presença de CTAB, $k_{\text {obs }}$ aumentou de $3,10 \times 10^{-2}$ a $3,31 \times 10^{-2} \mathrm{~s}^{-1}$, enquanto o aumento foi mais significativo, isto é, de $2,36 \times 10^{-2}$ a $3,79 \times 10^{-2} \mathrm{~s}^{-1}$ na ausência do surfactante, como pode ser observado na Figura 5a. A proporção de metanol é representada em termos da constante dielétrica, $\varepsilon$ (AKERLOF, 1932) nesta figura. Por outro lado, a constante de velocidade permaneceu praticamente constante tanto, na presença de CTAB, $3,64 \times 10^{-2}$ a $3,76 \times 10^{-2} \mathrm{~s}^{-1}$, quanto na ausência, ou seja, de $3,03 \times 10^{-2} \mathrm{a}$ $3,11 \times 10^{-2} \mathrm{~s}^{-1}$ (Figura 5b), no meio com força iônica controlada através da adição de $\mathrm{NaHSO}_{4}\left(0,35 \mathrm{~mol} \mathrm{~L}^{-1}\right)$.
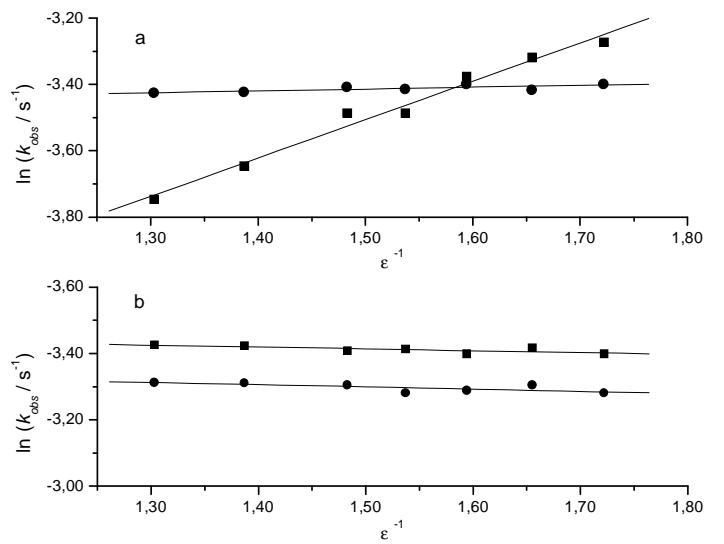

Figura 5 - Efeito do metanol na oxidação de ácido mandélico a $303 \mathrm{~K}$ na ausência $(\bullet)$ e na presença $(\bullet)$ de CTAB para forças iônicas iguais a $0,11 \mathrm{~mol} \mathrm{~L}^{-1}$ (a) e $0,35 \mathrm{~mol} \mathrm{~L}^{-1}$ (b) para [HMd] $0,50 \mathrm{~mol} \mathrm{~L}^{1},[\mathrm{~V}(\mathrm{~V})]_{\mathrm{o}} 1,00 \times 10^{-2} \mathrm{~mol} \mathrm{~L}^{-1} \mathrm{e}\left[\mathrm{H}_{2} \mathrm{SO}_{4}\right]_{0} 0,10 \mathrm{~mol} \mathrm{~L}^{-1}$.

\section{Efeito da Temperatura}

O efeito da temperatura na constante de velocidade foi investigado, variando-se de 298 a 318K. Para cada temperatura, a concentração inicial de ácido mandélico foi variada de 0,25 a 1,25 $\mathrm{mol} \mathrm{L}^{-1}$, mantendo-se constantes, $[\mathrm{V}(\mathrm{V})]_{\mathrm{o}} 1,00 \times 10^{-2} \mathrm{~mol} \mathrm{~L}^{-1}$, $\left[\mathrm{H}_{2} \mathrm{SO}_{4}\right]_{0} 0,10 \mathrm{~mol} \mathrm{~L}^{-1}$, na ausência e na presença de CTAB $2,5 \times 10^{-2} \mathrm{~mol} \mathrm{~L}^{-1}$. As constantes de velocidade de pseudo $1^{\text {a }}$. ordem foram determinadas para todas as concentrações de HMd (Tabela 2). A ordem de reação com relação a [HMd] permaneceu praticamente constante em $0,7(r=0,997)$ na ausência e de $0,6(r=0,997)$ na presença de CTAB. As constantes de velocidade de $2^{\mathrm{a}}$ ordem, $k_{2}$, foram calculadas a partir da inclinação da reta entre $k_{o b s}$ versus [HMd]. As energias de ativação foram determinadas pela equação de Arrhenius e equivalentes a 62,5 e $39,8 \mathrm{~kJ} \mathrm{~mol}^{-1}$ respectivamente na ausência e na presença de CTAB (Figura 6). As entropias de ativação foram calculadas baseando-se na equação de Eyring (ATKINS; DE PAULA, 2002) e resultaram em -76 e $-152 \mathrm{~J}^{\text {mol K}}{ }^{-1}$. 
Tabela 2 - Efeito da temperatura na oxidação de ácido mandélico por $\mathrm{V}(\mathrm{V})$ na ausência e presença de surfactante: $[\mathrm{HMd}]_{\mathrm{o}}=0,25-$ $1,25 \mathrm{~mol} \mathrm{~L}^{-1},[\mathrm{~V}(\mathrm{~V})]_{\mathrm{o}}=1,0 \times 10^{-2} \mathrm{~mol} \mathrm{~L}^{-1},\left[\mathrm{H}_{2} \mathrm{SO}_{4}\right]_{\mathrm{o}}=0,10 \mathrm{~mol} \mathrm{~L}^{-1}$.

\begin{tabular}{cccccccc}
\hline & & \multicolumn{5}{c}{$k_{\text {obs }} / 10^{-2} \mathrm{~s}^{-1}$} \\
\cline { 4 - 7 } $\mathrm{T} / \mathrm{K}$ & {$[\mathrm{CTAB}] / 10^{-2} \mathrm{~mol} \mathrm{~L}^{-1}$} & {$[\mathrm{HMd}] / \mathrm{mol} \mathrm{L}^{-1}$} & 0,25 & 0,50 & 0,75 & 1,00 & 1,25 \\
\hline 298 & 0 & 1,07 & 1,74 & 2,31 & 2,75 & 3,05 \\
303 & 0 & 1,49 & 2,36 & 3,26 & 3,87 & 4,70 \\
308 & 0 & 2,21 & 3,72 & 4,86 & 5,82 & 6,94 \\
313 & 0 & 3,60 & 5,52 & 7,98 & 9,43 & 9,61 \\
318 & 0 & 4,62 & 7,65 & 10,80 & 13,27 & 14,54 \\
298 & 2,5 & 1,71 & 2,53 & 3,01 & 3,41 & 3,80 \\
303 & 2,5 & 2,22 & 3,10 & 3,68 & 4,20 & 4,78 \\
308 & 2,5 & 2,65 & 3,92 & 4,96 & 5,49 & 6,32 \\
313 & 2,5 & 2,98 & 4,79 & 5,80 & 6,87 & 7,73 \\
318 & 2,5 & 3,46 & 5,22 & 6,59 & 7,74 & 8,72 \\
\hline
\end{tabular}

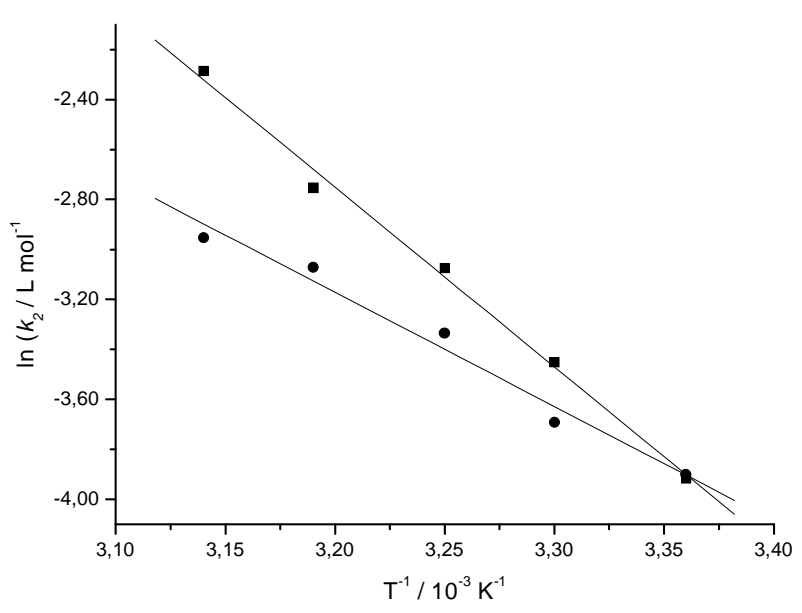

Figura 6 - Determinação dos parâmetros de ativação na ausência

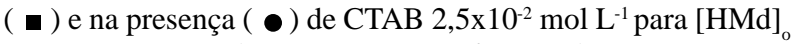
0,25 a $1,25 \mathrm{~mol} \mathrm{~L}^{-1},[\mathrm{~V}(\mathrm{~V})]_{\mathrm{o}} 1,00 \times 10^{-2} \mathrm{~mol} \mathrm{~L}^{-1}$ e $\left[\mathrm{H}_{2} \mathrm{SO}_{4}\right]_{\mathrm{o}} 0,10$ $\mathrm{mol} \mathrm{L} \mathrm{L}^{-1}$.

\section{Discussão}

A lei de velocidade empírica para a oxidação de ácido mandélico possui ordem fracionária tanto em relação à concentração de ácido mandélico (Figura 2), quanto à de ácido sulfúrico (Figura 3), mantendose ou não a força iônica. A oxidação foi mais rápida no meio contendo o surfactante catiônico CTAB, se comparado ao meio sem este. A reação é de primeira ordem individualmente com relação à concentração de vanádio(V), mas a constante de velocidade diminui com o aumento da concentração de vanádio(V) no intervalo de $0,5 \times 10^{-2}$ a $2,5 \times 10^{-2} \mathrm{~mol} \mathrm{~L}^{-1}$ na ausência de surfactante. Na presença de CTAB, as constantes de velocidade são, pelo menos, $30 \%$ maiores, mas há a peculiaridade de diminuir a constante de velocidade de $3,24 \times 10^{-2} \mathrm{~s}^{-1} \mathrm{em} 0,5 \times 10^{-2} \mathrm{~mol} \mathrm{~L}^{-1}$ até $3,04 \times 10^{-2} \mathrm{~s}^{-1}$ em $1,5 \times 10^{-2} \mathrm{~mol} \mathrm{~L}^{-1} \mathrm{e}$ aumentar para $3,38 \times 10^{-2} \mathrm{~s}^{-1} \mathrm{em}$ $2,5 \times 10^{-2} \mathrm{~mol} \mathrm{~L}^{-1}$ (Figura 2). A diminuição da constante de velocidade tem sido atribuída ao fato de o vanádio(V) reagir como espécie coordenada $\mathrm{V}(\mathrm{OH})_{3} \mathrm{HSO}_{4}{ }^{+} \quad$ ou $\quad \mathrm{V}(\mathrm{OH})_{2}\left(\mathrm{HSO}_{4}\right)_{2}{ }^{+}$ (TAKASHIMA; ZIGLIO; RONCONI, 1998). Isto dificulta a interação com o ácido mandélico que possui um grupo fenila em sua estrutura, resultando em impedimento estérico. Por outro lado, o aumento observado na constante de velocidade após a concentração de $1,5 \times 10^{-2} \mathrm{~mol} \mathrm{~L}^{-1}$, sugere que o surfactante forma com o oxidante e o substrato uma espécie mais reativa. A lei de velocidade empírica é então dada por:

$$
v=-\frac{d[V(V)]}{d t}=k_{o b s}[H M d]^{x}\left[H^{+}\right]^{y}[V(V)]
$$

independentemente da força iônica, na qual $x$ e $y$ representam as ordens fracionárias respectivamente para as concentrações de ácido mandélico e íon hidrogênio.

Entre os $\alpha$-hidroxiácidos investigados por vanádio(V) em meio ácido, o ácido mandélico é o mais rapidamente oxidado, indicando que o grupo aromático localizado na posição $\alpha$ aumenta a 
velocidade de oxidação. Isto pode ser verificado experimentalmente pelas menores concentrações de ácido sulfúrico $\left(0,5 \times 10^{-1}\right.$ a $\left.3,0 \times 10^{-1} \mathrm{~mol} \mathrm{~L}^{-1}\right)$ e maiores constantes de velocidade tanto na ausência $\left(1,65 \times 10^{-}\right.$ 2 a $\left.4,24 \times 10^{-2} \mathrm{~s}^{-1}\right)$ quanto na presença do surfactante CTAB $\left(2,41 \times 10^{-2}\right.$ a $\left.5,51 \times 10^{-2} \mathrm{~s}^{-1}\right)$, como podem ser observadas na Figura 3. Em ambos os casos a ordem de reação permaneceu com valor fracionário, ou seja, $0,52 \mathrm{em}$ meio sem surfactante e 0,46 na presença de CTAB.

Assim como nas oxidações anteriores estudadas, é observada a dependência linear no gráfico entre $\log k_{o b s}$ contra a raiz quadrada da força iônica. Os produtos iônicos, $Z_{+} Z$, equivalentes a 0,54 e 0,43 foram obtidos respectivamente na ausência e na presença

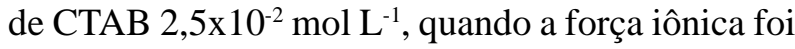
variada de 0,11 a $1,00 \mathrm{~mol} \mathrm{~L}^{-1}$ (Figura 4). A partir apenas do produto iônico, é difícil sugerir se o vanádio $(\mathrm{V})$ reagiria com o ácido mandélico na forma protonada ou molecular. A constante de velocidade foi ao redor de $30 \%$ maior, quando se adicionou $\mathrm{CTAB}$ de $1,0 \times 10^{-2}$ a $10,0 \times 10^{-2} \mathrm{~mol} \mathrm{~L}^{-1}$ para a força iônica fixada em $0,35 \mathrm{~mol} \mathrm{~L}^{-1}$ a $303 \mathrm{~K}$ com relação àquela sem controle $\left(0,11 \mathrm{~mol} \mathrm{~L}^{-1}\right)$ mantendo-se praticamente inalterada na presença e na ausência deste eletrólito (Tabela 1). A partir destes resultados foi possível concluir que, a contribuição do surfactante é um pouco mais significativa que a do eletrólito na constante de velocidade. Além disso, o aumento linear de $\ln k_{o b s}$ em função do inverso da constante dielétrica (AKERLOF, 1932) em solução sem controle de força iônica na presença de surfactante, sugere que o ácido mandélico parece não estar protonado em metanol e interage com a espécie positivamente carregada de vanádio(V). Quando a variação de ln da constante de velocidade é mais acentuada na ausência de surfactante, o $\alpha$-hidroxiácido parece adquirir carga positiva e aumentar a velocidade de reação ao reagir com a espécie positivamente carregada de vanádio(V) (Figura 5).

O mecanismo é similar em ambos os meios, com e sem surfactante, porque a ordem da reação permaneceu constante, quando a temperatura foi variada de 298 a $318 \mathrm{~K}$, segundo os resultados mostrados na Tabela 2. A diminuição da energia de ativação $\left(39,8 \mathrm{~kJ} \mathrm{~mol}^{-1}\right)$ na presença de CTAB indica que a oxidação é mais rápida do que em meio sem CTAB $\left(62,5 \mathrm{~kJ} \mathrm{~mol}^{-1}\right)$ (Figura 6). O valor negativo para a entropia de ativação é indicativo para um fator de frequiência mais baixo e de formação de complexos ativado mais rígido do que os reagentes. Em meio de CTAB a entropia de ativação foi de $-152 \mathrm{~J} \mathrm{~mol}^{-1} \mathrm{~K}^{-1}$ enquanto que na ausência, $-76 \mathrm{~J} \mathrm{~mol}^{-1} \mathrm{~K}^{-1}$. Isto é atribuído à ligação das moléculas de solvente no complexo ativado mais fortemente do que as moléculas de reagentes (MOORE; PEARSON, 1981).

Apesar de a constante de velocidade de oxidação de ácido mandélico em meio de CTAB ser maior, a reação não ocorre apenas pela catálise micelar, devido à solubilidade do substrato em água, prejudicando a mobilidade das moléculas de surfactante e a interação das micelas, supostamente formadas com o complexo ativado.

A partir dos resultados obtidos, é proposto o mecanismo de oxidação do ácido mandélico por vanádio (V) em meio ácido, constituído pelas seguintes etapas:

$$
\begin{aligned}
& \mathrm{V}(\mathrm{OH})_{3} \mathrm{HSO}_{4}^{+}+\mathrm{H}^{+}+\mathrm{C}_{6} \mathrm{H}_{5} \mathrm{CH}(\mathrm{OH}) \mathrm{CO}_{2} \mathrm{H} \stackrel{K_{2}}{\stackrel{X_{2}}{\longrightarrow}} \mathrm{X}^{* 2+} \\
& \mathrm{X}^{* 2+} \underset{k_{2}}{\longrightarrow} \mathrm{C}_{6} \mathrm{H}_{5} \mathrm{CH} \bullet(\mathrm{OH})+\mathrm{V}(\mathrm{OH})_{2} \mathrm{HSO}_{4}^{+}+\mathrm{CO}_{2}+\mathrm{H}^{+} \\
& \mathrm{V}(\mathrm{OH})_{3} \mathrm{HSO}_{4}^{+}+\mathrm{C}_{6} \mathrm{H}_{5} \mathrm{CH} \bullet(\mathrm{OH}) \stackrel{k_{3}}{\longrightarrow} \\
& \mathrm{V}(\mathrm{OH})_{2} \mathrm{HSO}_{4}^{+}+\mathrm{C}_{6} \mathrm{H}_{5} \mathrm{COH}+\mathrm{H}_{2} \mathrm{O}
\end{aligned}
$$

Assumindo que $k_{2}$ seja a etapa determinante da reação, a lei de velocidade é dada por:

$$
v=\frac{k_{2} K_{1}[H M d]\left[H^{+}\right][V(V)]_{t}}{1+K_{1}[H M d]\left[H^{+}\right]}=k_{o b s}[V(V)]_{t}
$$

em que $[\mathrm{V}(\mathrm{V})]_{\mathrm{t}}=\left[\mathrm{X}^{*+}\right]+\left[\mathrm{V}(\mathrm{OH})_{3} \mathrm{HSO}_{4}^{+}\right]$e a constante de velocidade de pseudo- $1^{\mathrm{a}}$. ordem dada por:

$$
k_{o b s}=\frac{k_{2} K_{1}[H M d]\left[H^{+}\right]}{1+K_{1}[H M d]\left[H^{+}\right]}
$$

em concordância com a lei de velocidade empírica. 


\section{Conclusão}

A partir desses resultados, pode-se concluir que o surfactante CTAB aumenta a velocidade de oxidação, embora, aparentemente não haja formação de micelas entre o ácido mandélico e o CTAB. Isso ocorre porque o efeito da força iônica na constante de velocidade é similar àquele causado pela adição de CTAB. A lei de velocidade de oxidação de ácido mandélico por $\mathrm{V}(\mathrm{V})$ em meio ácido na ausência e na presença de CTAB é de ordem fracionária, tanto com relação à concentração de ácido mandélico, quanto com relação ao íon hidrogênio. As energias de ativação calculadas indicam que a oxidação de ácido mandélico por vanádio(V) é mais rápida em meio de CTAB. As entropias de ativação indicam que o complexo ativado formado é mais compacto que os reagentes que o formam. $\mathrm{O}$ mecanismo de oxidação proposto está em concordância com os resultados experimentais.

\section{Agradecimentos}

Os autores (E.N. e R.A.A.) agradecem ao CNPq/ PIBIC e à PRO-PPG/UEL pelas bolsas de Iniciação Científica.

\section{Referências}

AKERLOF, G. Dielectric constants of some organic solvent-water mixtures at various temperatures. Journal of the American Chemical Society, Chicago, v.54, n.11, p.4125-4139, 1932.

ANDO, R. A.; RAMINELLI, C.; BARRETO, W. J.; TAKASHIMA, K. Oxidation of two $\alpha$-hydroxy acids by vanadium(V). Monatshefte Für Chemie, Wien, v.134, n.10, p.1321-1331, oct. 2003.

ATKINS, P. W.; DE PAULA, J. Physical Chemistry, 7 ed. Oxford: Oxford, 2002.
BRUYÈRE, V. I. E.; RODENAS, L. A. G.; MORANDO, P. J.; BLESA, M. A. Reduction of vanadium $(\mathrm{V})$ by oxalic acid in aqueous acid solutions. Journal of the Chemical Society- Dalton Transactions, Cambridge,v.24, p.3593-3597, 2001.

CLEMENTIN, R. M.; TAKASHIMA, K. Oxidation of lactic by vanadium(V) in sulfuric acid medium: a kinetic and mechanistic study. Química Nova, São Paulo, v.16, p.529-533, 1993.

DIN, K.; MORSHED, A. M. A.; KHAN, Z. Influence of sodium dodecyl sulfate/tritonX-100 micelles on the oxidation of D-fructose by chromic acid in presence of $\mathrm{HClO}_{4}$. Carbohydrate Research, Amsterdam, v.337, p.1573-1583, 2002.

KHAN, Z.; BABU, P. S. S.; DIN, K. Kinetics and mechanism of the oxidation of D-fructose by vanadium(V) in $\mathrm{H}_{2} \mathrm{SO}_{4}$ medium. Carbohydrate Research, Amsterdam., v.339, p.133-140, 2004.

MEHROTRA, R. N. Studies of kinetic acidity dependence in certain oxidation reactions. Journal of the Chemical Society B-Physical Organic, London, v.6, p.642-644, 1968.

MEHROTRA, R. N. Kinetic acidity dependence in certain oxidation reactions. Part II. Oxidation of propane-1,3- and butane-1,4-diols by quinquevalent vanadium ion. Journal of the Chemical Society BPhysical Organic, London, p.1123-1127, 1968.

MOORE, J. W.; PEARSON, R. G. Kinetics and mechanism. 3. ed. New York: J. Wiley, 1981. p.255-257.

PANDEY, S.; UPADHYAY, S. K. Effect of cationic micellar aggregates on the kinetics of oxidation of aminoalcohols by $\mathrm{N}$-bromosuccinimide in alkaline medium. Journal of Colloid and Interface Science, New York, v.285, n.2, p.789-794, may 2005. 
RAMINELLI, C.; BARRETO, W. J.; TAKASHIMA,

K. Citric acid oxidation by vanadium in sulfuric acid medium. International Journal of Chemical Kinetics, New York, v.32, n.9, p.566-572, sep. 2000.

TAKASHIMA, K.; ZIGLIO, C. M.; RONCONI, C. M. Kinetic and mechanistic aspects for the tartaric acid oxidation by vanadium(V) in sulfuric acid medium. International Journal of Chemical Kinetics, New York, v.30, n.1, p.55-61, jan. 1998.

TAKASHIMA, K. Kinetics and mechanism of malic acid oxidation by vanadium(V) in acidic medium. Química Nova, São Paulo, v.16, p.409-413, 1993.

ZIGLIO, C. M.; TAKASHIMA, K. Oxidation of malic acid by vanadium $(\mathrm{V})$ in sulphuric acid medium: a kinetic and mechanistic investigation. International Journal of Chemical Kinetics, New York, v.27, n.11, p.1055-1064, nov. 1995. 